OPEN ACCESS

Edited by:

Paulo Henrique Rosado-de-Castro, Instituto D'Or de Pesquisa e Ensino

(IDOR), Brazil

Reviewed by:

Yuka lkegame,

Kizawa Memorial Hospital, Japan Catriona Cunningham,

University of Manchester,

United Kingdom

*Correspondence:

María Gutiérrez-Fernández

mgutierrezfernandez@

salud.madrid.org

tThese authors have contributed equally to this work

Specialty section This article was submitted to Stroke,

a section of the journa

Frontiers in Neurology

Received: 30 November 2018 Accepted: 19 March 2019 Published: 09 April 2019

Citation:

Laso-García F, Diekhorst L, Gómez-de Frutos MC, Otero-Ortega L, Fuentes B, Ruiz-Ares $G$ Díez-Tejedor E and Gutiérrez-Fernández M (2019) Cell-Based Therapies for Stroke: Promising Solution or Dead End?

Mesenchymal Stem Cells and Comorbidities in Preclinical Stroke Research. Front. Neurol. 10:332. doi: 10.3389/fneur.2019.00332

\section{Cell-Based Therapies for Stroke: Promising Solution or Dead End? Mesenchymal Stem Cells and Comorbidities in Preclinical Stroke Research}

\author{
Fernando Laso-García ${ }^{\dagger}$, Luke Diekhorst ${ }^{\dagger}$, Mari Carmen Gómez-de Frutos, \\ Laura Otero-Ortega, Blanca Fuentes, Gerardo Ruiz-Ares, Exuperio Díez-Tejedor and \\ María Gutiérrez-Fernández*
}

Neuroscience and Cerebrovascular Research Laboratory, Department of Neurology and Stroke Center, La Paz University Hospital, Neuroscience Area of IdiPAZ Health Research Institute, Autonoma University of Madrid, Madrid, Spain

Stroke is a major health problem worldwide. It has been estimated that $90 \%$ of the population attributable risk of stroke is due to risk factors such as aging, hypertension, hyperglycemia, diabetes mellitus and obesity, among others. However, most animal models of stroke use predominantly healthy and young animals. These models ignore the main comorbidities associated with cerebrovascular disease, which could be one explanation for the unsuccessful bench-to-bedside translation of protective and regenerative strategies by not taking the patient's situation into account. This lack of success makes it important to incorporate comorbidities into animal models of stroke in order to study the effects of the various therapeutic strategies tested. Regarding cell therapy, the administration of stem cells in the acute and chronic phases has been shown to be safe and effective in experimental animal models of stroke. This review aims to show the results of studies with promising new therapeutic strategies such as mesenchymal stem cells, which are being tested in preclinical models of stroke associated with comorbidities and in elderly animals.

Keywords: aging, hypertension, diabetes, hyperglycemia, obesity, comorbidity, mesenchymal, stroke

\section{INTRODUCTION}

Stroke is still the most common cause of permanent disability in adults and the second leading cause of death in the world (1). The pathology of stroke is poorly understood; however, it has been shown that the majority of patients with stroke have at least one comorbidity (2). The contribution of various risk factors to worldwide stroke burden is unknown. The INTERSTROKE study has demonstrated that five risk factors accounted for more than $80 \%$ of the global risk for all strokes (either ischemic stroke or intracerebral hemorrhage [ICH]): hypertension, current smoking, abdominal obesity, diet and physical activity (3). Furthermore, stroke incidence rises with increased in age (4). This high prevalence of comorbidities in stroke patients indicates the need for therapies in preclinical studies that take these comorbidities into account (Figure 1).

Of 502 experimental therapies for acute focal ischemic stroke, only $10 \%$ were tested in animals with hypertension. Hypertensive animals have larger infarct sizes and reduced efficacy with 


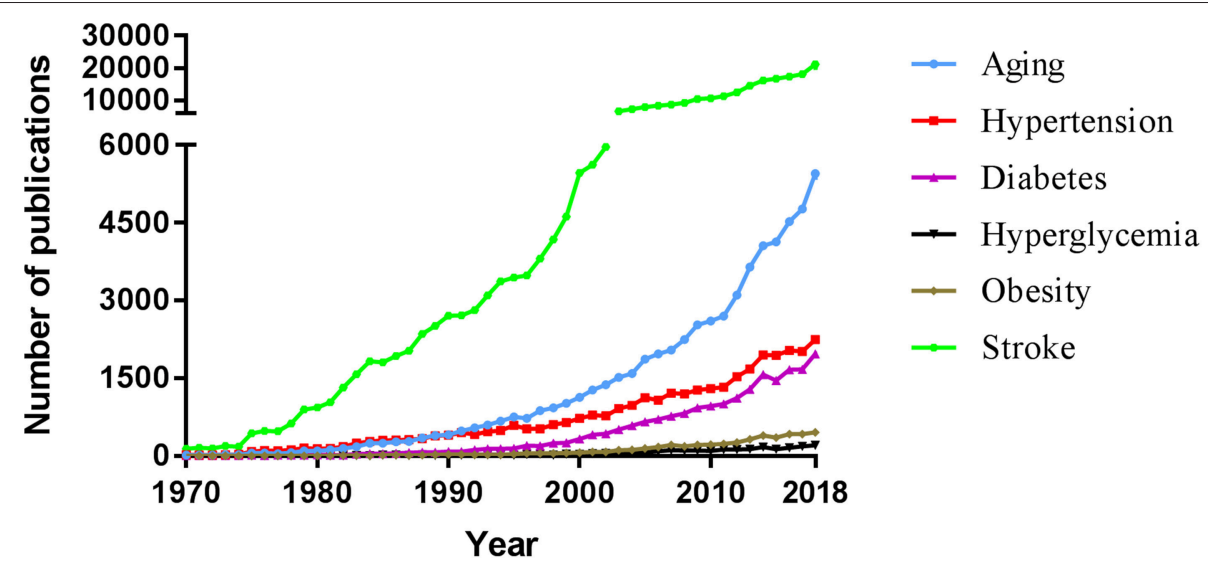

FIGURE 1 | Comparative evolution of the evaluation of the effect of various comorbidities in animal models of stroke as well as in clinical research. An advanced search was performed in PubMed in December 2018 to find, for each year of publication, all articles using the text word stroke with the given term as text word: age or aging; hypertension or hypertensive or high blood pressure; diabetes or diabetes mellitus; hyperglycemia or hyperglycemic or high blood sugar or high glucose levels; or obesity or overweight. Year of final publication (and not advanced online date) of articles in English (and not other languages) was taken into account. Manual elimination of articles describing a non-comorbid stroke association were excluded.

therapeutic intervention $(5,6)$. Even fewer preclinical studies assess the effects of diabetes or acute hyperglycemia on the response to therapeutic intervention (7). The majority of preclinical studies for novel therapies use young healthy animal models and this may play a role in the fact that of 1,026 treatments tested on animal models, only one has been effective in clinical trials (8).

In particular, stem cell therapy has been proven to be effective mostly in healthy animals. Various types of stem cells have been used in preclinical stroke models: embryonic stem cells, neural stem cells, induced pluripotent stem cells, mesenchymal stem cells (MSCs) and hematopoietic stem cells (9). Cell therapy has been shown to promote functional recovery, participating in processes such as immunomodulation, neurogenesis, synaptogenesis, oligodendrogenesis, axonal connectivity, and myelin formation, improvement in blood brain barrier (BBB) integrity, neovascularization and reduced lesion size, showing efficacy not only in grey matter, but also white matter injury (10-17). However, its mechanisms of action has not yet been clarified. Recent evidence has suggested that it might be related to long-distance cell-to-cell communication by paracrine function through secretory factors in the extracellular environment. Intercellular communication between stem cells and the damaged organ was thought to be regulated via the release of free molecules that transmit the signal by binding to a receptor. These molecules could in part be trophic factors, inflammation modulators and even exosomes. In order to avoid previous translation failure in stem cell therapy, STAIR guidelines suggest that further studies should be performed on animals with comorbid conditions such as hypertension and

Abbreviations: ICH, intracerebral hemorrhage; MSCs, mesenchymal stem cells; BBB, blood-brain barrier; BMMNCs, bone marrow mononuclear cells; BMSCs, bone marrow stromal cells; G-CSF, granulocyte colony-stimulating factor; BMMSCs, bone marrow mesenchymal stem cells; SHRs, spontaneously hypertensive rats; SHR-SPs, stroke-prone SHRs; HUCBCs, human umbilical cord blood cells; DM-BMSCs, BMSCs derived from type I diabetes rats. diabetes in order to improve the quality of preclinical studies of purported stroke therapies (18).

This review is focused on MSC therapies being tested in preclinical models of stroke with the most common comorbidities (hypertension, hyperglycemia, diabetes, obesity), as well as in elderly animals. We intend to provide insight into the viability of this new strategy, which could lead to an improved translation of cell therapy from bench to bedside.

\section{MESENCHYMAL STEM CELLS IN PRECLINICAL STUDIES}

\section{Aging in Stroke}

Age is the most important risk factor for developing a stroke. Age has been proven to be a predictive factor for recovery after stroke, independent of stroke severity, characteristics and complications (19). Stroke incidence is also strongly but not solely correlated with an increase in age, in addition to the patient's general fitness (4). As previously stated, patient data shows a direct correlation between age and the occurrence of stroke. For these reasons, the impact of age should be considered carefully in preclinical studies given that the mechanisms of stroke and response to drugs can be very different in the developing, juvenile, adult and elderly brain (20). However, despite being the most important risk factor, there are currently few studies (only 7) on aged animals that evaluate the effect of administering MSC after ischemic stroke (Table 1). In this regard, systemic administration of bone marrow mononuclear cells (BMMNCs) in the acute phase after stroke reduced neurological deficits $(21,22)$ and reduced infarct volume, modulating post-ischemic inflammatory cytokines within the brain in older rats (21). Also in the acute phase, intravenous administration of human umbilical tissue-derived cells improved recovery of neurological function in aged rats after stroke and was associated with activation of repair processes (23). This beneficial effect of cell therapy is not only observed in the short 
term, but also in the long term. In one study, intra-arterial administration of bone marrow stromal cells (BMSCs) at 1 day after ischemic stroke had long-lasting beneficial effects on recovery of neurological functional (24). One interesting strategy for reducing ischemic damage to the brain would be based on a combination of therapies to act in different steps of the ischemic cascade. In this sense, the combination the granulocyte colonystimulating factor (G-CSF) with bone marrow mesenchymal stem cells (BMMSCs) increased neurogenesis and improved microvessel recovery and density in the aged brain (25). However, another study demonstrated that the combination of G-CSF and bone marrow mononuclear cells (BMMNCs) did not further improve post-stroke recovery (26).

Currently, all studies with MSCs in elderly animals have been performed on ischemic stroke, none on hemorrhagic stroke. Also, no standardized protocols were used in the above cell therapy studies for observing the various routes of administration and doses used. The number of studies is still very limited and further preclinical research is needed to determine the efficacy of MSCs in aged animal models. In view of the results, however, the aged brain retains the capacity for repair in response to cell therapy.

\section{Hypertension in Stroke}

Hypertension is considered one of the most common and important vascular risk factors for stroke (3) and is responsible for approximately $52 \%$ of strokes (5); it is also closely correlated with stroke severity. Hypertension has numerous effects, such as reducing $\mathrm{BBB}$ integrity and promoting white matter damage and post-stroke edema (5).

In order to test a new therapeutic strategy, a good experimental animal model must first be selected. Several models have been used to induce hypertension in animals. In the past, dogs were used in experiments as a hypertension model. Currently, the rat has become the common model for research as a cost-effective alternative. There are various ways to induce hypertension in rats; for example, spontaneously hypertensive rats (SHRs), stroke-prone SHRs (SHR-SPs), endocrine hypertension by deoxycorticosterone acetate administration, angiotensin II administration and hypertension induced by stress (47-49). Based on consistent reproducibility, the SHR is probably the best model with which to observe hypertension.

Regarding cell therapy, MSCs have been used in hypertensive rats to evaluate the efficacy of functional recovery in animals (Table 1).

In cerebral ischemia, intracerebral transplantation of BMMSCs has been shown to decrease apoptotic neurons in the neocortex and ameliorate brain damage by decreasing cell death (27). This has been shown to play a primary role in brain protection. BMMSCs also act on vasculogenesis in SHR rats. Thus, the cells significantly increased the number of microvessels and their reactivity to collagen IV in the neocortex, which indicates protection of the neurovascular unit and improvement of vascular integrity (27). In this regard, BMMSCs also increase of levels of the antiapoptotic B-cell lymphoma 2 (Bcl-2) gene and decrease superoxide, demonstrating that MSC has antioxidant potential and a protective effect in SHR rats with stroke (28).
Also, intravenously administered dual transplantation of human maternal or fetal placenta MSCs produces increased density of glial fibrillary acidic protein-positive cells in the area adjacent to the infarct border which may increase survival rates of regenerative astrocytes, leading to a decrease in infarct volume on day 60, triggering functional improvement in SHRs (29). However, not all studies have reported favorable functional outcomes after ischemic stroke with hypertension SHRs. BMMNCs or cryopreserved human umbilical cord blood mononuclear cells given intravenously did not show a beneficial effect on infarct volume, behavioral outcomes or inflammatory response $(30,31)$. Pösel et al. performed a study to determine a possible synergistic effect of G-CSF and BMMNCs after stroke in SHR rats, in which they found administration of G-CSF improved long-term functional recovery. However, this effect was negated by cotransplantation of BMMNCs as provoked splenic accumulation of granulocytes and transplanted cells, accompanied by a significant rise in circulating granulocytes and infiltration in the ischemic brain, which was detrimental to stroke outcome (32).

In addition, the Framingham Study clearly demonstrated the relevance of age and high blood pressure for lifetime risk of stroke (50), indicating the need to mimic these risk factors in preclinical stroke studies. Along these lines, animals transplanted with intravenous bone marrow cells from young SHR-SPs displayed an increase in microvasculature density in the periinfarction zone which led to reduced ischemic brain damage and improved neurological function (33). However, in the same study BM cells led to a significant increase in levels of cytokines such as interleukin $1 \beta$ (IL-1 $\beta$ ) and monocyte chemoattractant protein 1 (MCP-1) in the brain and a decrease of IL- 6 levels in serum. These results suggest that modulation in the expression of inflammatory cytokines (i.e., favoring recovery/decreased inflammatory profile) did not occur and, therefore, is not likely to explain the beneficial effect of the response to cerebral ischemia observed in older SHR-SPs transplanted with BM cells from young SHR-SPs (33). Additionally, further studies reported negative results, such as the study by Wagner et al. which evaluated the therapeutic efficacy of intravenously transplanted young and aged BMMNCs in aged hypertensive rats. The authors concluded that BMMNCs from both juvenile and elderly donors failed to decrease lesion volume and functional recovery was not improved (34).

There are currently two studies in SHRs with ICH. In both studies, intravenously (35) or intracerebrally (36) transplanted BMMSCs improved neurological function and integrity of the $\mathrm{BBB}$ by preventing extravasation of blood through the endothelium $(35,36)$, resulting in improvements such as reduced brain edema and decreased cell apoptosis (36).

Although several different doses and administration routes have been used for treatment in hypertensive animals poststroke, contradictory results have been found between different research groups using MSCs in hypertensive animals (Table 1). More studies should be performed to evaluate whether MSC therapy is effective not only in brain protection, but also in brain repair in the treatment of stroke in hypertensive animals. 
TABLE 1 | Original studies evaluating the effect of MSC administration in ischemic stroke models using aged animals, hypertension- and diabetes-induced stroke models.

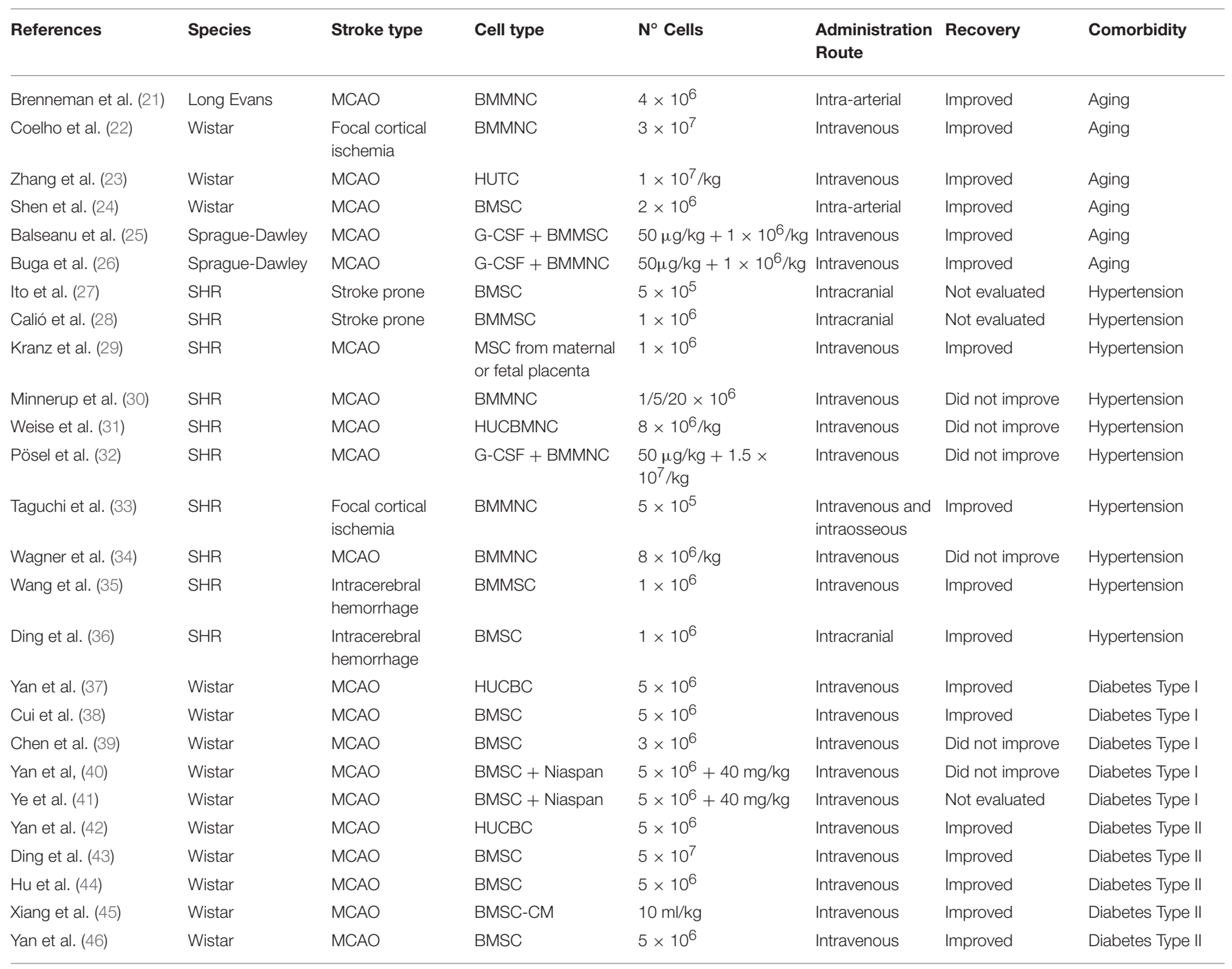

MCAO, middle cerebral artery occlusion; BMMNC, bone marrow mononuclear cell; HUTC, human umbilical tissue-derived cell; BMSC, bone marrow stromal cell; G-CSF, granulocyte colony-stimulating factor; BMMSC, bone marrow mesenchymal stem cell; SHR, spontaneously hypertensive rats; G-CSF, granulocyte colony-stimulating factor; HUCBMNC, human umbilical cord blood mononuclear cell; HUCBC, human umbilical cord blood cell; BMSC-CM, bone marrow stromal cell conditioned medium.

\section{Diabetes in Stroke}

Diabetes is divided into two types: type 1, in which the beta-cells of the pancreas are damaged and affected people need external administration of insulin; and type 2 , which is peripheral insulin resistance, and is present in $85 \%$ of the patients with diabetes $(51,52)$. Diabetes causes several metabolic and pathological changes that lead to stroke including arterial stiffness, systematic inflammation, endothelial dysfunction and heart failure (53). In addition, stroke in diabetes patients increases hospital mortality (54).

There are many methods to try to mimic type 1 and 2 diabetes in rats (55). One of the most commonly used models for diabetes type 1 are the Biobreeding rats; these rats develop diabetes spontaneously or it is induced by a virus (55). Recently, however, injection of chemicals to destroy beta-cells in the islets of Langerhans has been growing in importance.
Alloxan has been used for some time as a good diabetic model but it has problems such as spontaneous recovery from the diabetic condition or renal toxicity. To solve this, another beta-cytotoxic agent, streptozotocin, is used. One advantage of this chemical is that the damage is dose-dependent, which allows researchers to control the severity of that animals' hyperglycemia (56).

To replicate type 2 diabetes, various spontaneous models are used in laboratories such as the spontaneously diabetic tori rat (57), due to gradual beta-cell degeneration, or GotoKakizaki rats, which develop peripheral insulin resistance after 56 days (58). Another option is induction by a high fat diet and intraperitoneal streptozotocin administration, in which the rats develop hyperinsulinemia, obesity and a reduction in beta-cells (59). In addition, administration of nicotinamide intraperitoneally prior to administration of a low dose of 
streptozotocin protects the cells by attenuating the effect of streptozotocin (60).

Regarding MSC therapy, human umbilical cord blood cells (HUCBCs) and BMSCs have been shown to contribute to an increase in phosphorylated neurofilament marker SMI-31 and synaptophysin expression. These markers are involved in axonal and synaptic plasticity that promotes white matter remodeling in the ischemic brain $(37,38,41)$. Vascular remodeling was revealed by an increase in the expression of smooth muscle actin $(\alpha-$ SMA) and Von Willebrand Factor (vWF) (37) in the ischemic brain which led to an improvement in functional outcomes (37, 38). In addition, BMSCs decreased miR-145 expression, which reduces endothelial cell proliferation, contributing to increased functional cells and restorative effects in type 1 diabetic rats (38). However, contradictory results have been reported by other authors. BMSC treatment by tail vein starting $24 \mathrm{~h}$ after middle cerebral artery occlusion in diabetes type 1 rats resulted in increased brain hemorrhage, BBB leakage and higher expression of angiogenin. This causes accelerated cerebral arteriosclerosis and prevents improvement in functional outcomes (39).

In subsequent studies, however, the harmful effects of BMSC administration were negated when the treatment was administered in combination with Niaspan. Despite the combination, BMSC and Niaspan treatment for stroke did not improve functional outcomes. However, it did decrease BBB leakage and atherosclerotic-like changes (40) and promoted white matter remodeling in type 1 diabetes rats after stroke (41) (Table 1).

Regarding type 2 diabetes, all experimental animal studies have shown a beneficial effect of MSCs. Independent of treatment with HUCBCs, BMSCs or bone marrow stromal cellconditioned medium initiated at $24 \mathrm{~h}$ or 3 days after stroke via intravenous administration improved functional recovery, promoted restorative effects and reduced $\mathrm{BBB}$ disruption after stroke in type 2 diabetes rats (42-46) (Table 1). As in type 1 diabetes, MSC therapy is also associated with white matter remodeling in type 2 diabetes $(42,46)$, participating in axonal regeneration, sprout and remyelination which led to improved long-term functional outcomes (46). MSC therapy after stroke also contributes to vascular remodeling in type 2 diabetes. Specifically, BMSC-CM treatment enhanced expression of angiopoietin 1 (Ang1), tyrosine-protein kinase receptor Tie2 (45), $\alpha$-SMA, and $\operatorname{vWF}(39,43)$, which indicates higher cerebral artery and vascular density (42). Ang1 also seems to be related with a reduction in $\mathrm{BBB}$ leakage and promotes vascular stabilization in the ischemic brain. Moreover, it plays a role in white matter remodeling $(37,41)$, which may improve functional outcome. Regarding the immune system, several authors defend the idea that MSC treatment can also regulate pro-inflammatory factors. This has been shown by a decrease in expression of the receptor for advanced glycation end-products (RAGE) after HUCBC (42) and BMSC (44) treatment in diabetic rats. This indicates a decrease in inflammation, neuronal death, vascular injury and brain damage following ischemia in type 2 diabetic rats. HUCBC and BMSC treatment of type 2 diabetic stroke rats also had an effect on macrophage polarization, promoting decreases inflammation $(42,46)$, decreased the expression of the proinflammatory protein toll-like receptor 4 (TLR4) (42), increasing brain platelet-derived growth factor (PDGF) expression in the ischemic brain, contributing to restoration (46) and promoting functional improvement after stroke (42).

In summary, with regard to type 1 diabetes, although the experimental studies are homogeneous in terms of the route of administration, cell dose and stroke location, the results are contradictory with no good functional recovery observed in any of them. This reveals the need for further research to increase understanding of the interaction between type 1 diabetes mellitus and cell-based therapy with MSCs. Although all the studies performed thus far reveal that treatment of type 2 diabetes with MSCs in ischemic stroke models can be successful, to our knowledge no studies have been conducted to test the efficacy of MSCs in hemorrhagic stroke. The meager interest aroused could be due to the fact that the prevalence of diabetes is higher in patients with ischemic compared with hemorrhagic stroke, as recently demonstrated by a meta-analysis (61).

\section{Hyperglycemia in Stroke}

Hyperglycemia plays an important role in stroke and is associated with poorer functional recovery and an increase in mortality in ischemic and hemorrhagic stroke $(62,63)$. It has been observed that after stroke, hyperglycemia is present in over $50 \%$ of patients (64). Given the high percentage of patients who develop poststroke hyperglycemia, it is important to conduct research in hyperglycemic animal models. Two options have been used to reproduce hyperglycemic situations in preclinical stroke models, depending on the researchers' requirements: acute hyperglycemia after anesthesia can be mimicked in animals with intravenous infusion of glucose (65); or intravenous administration of streptozotocin and continuous administration of insulin, in which the animals develop hyperglycemia after 1 week (66). To our knowledge, no data have been published on how MSC-based therapy affects stroke associated with hyperglycemia.

\section{Obesity in Stroke}

Obesity, especially in the abdominal zone (67), is an important risk factor at all ages. An increase in body mass index (BMI) significantly increases the risk of stroke. Compared with healthy weight individuals, the obese population has a $64 \%$ greater probability of experiencing an ischemic stroke (68). The association between BMI and ischemic stroke is linear, without differences between sex or race (69). Other studies also linked obesity with hemorrhage, in which people with a high BMI have a $37 \%$ higher incidence (70).

Various animal models have been established to induce obesity in rats (71). Yet despite the high prevalence of obesity in stroke, no research has been performed on how MSC therapy affects the pathology of stroke associated with obesity. In conclusion, studies should be conducted to show the interactions between obesity and MSC treatment after stroke.

\section{Take Home Message}

Most of the studies carried out on this subject indeed favor BMMSCs. However, compared to other cell types, adipose tissue-derived mesenchymal stem cells (ADMSCs) have 
several advantages in clinical applications for neurological disorders (72). ADMSCs are derived from adipose tissue and thus are abundant, accessible and easy to obtain using lipoaspiration techniques. Moreover, they provide proliferation and differentiation potential (73) without adverse side effects (12, $74,75)$ and they can be administered without ethical concerns. All of these advantages mean that ADMSCs present a great opportunity for the treatment of diseases such as comorbidities in stroke. Further studies should take this into consideration.

It should be emphasized that patients present not only one but also several associated comorbidities at a time. Therefore, it is clear that there is a need for multimodelling for successful translation of preclinical research to the clinic (6). In this sense, not only modifiable factors, but also non-modifiable risk factors such as age and sex, are important to include in animal model studies (6). Moreover, animal models with co-morbidities show higher variability in outcome measures and therefore, higher sample sizes should be estimated with the specific disease model in mind (6). Also, aged animals take longer to recover after stroke, but eventually recovered to the same degree as young mice, making clear the importance of implementing long-term studies (76).

Adequate selection of the experimental model for stroke and comorbidity induction is important to reduce mortality, as it is often higher in models with preexisting comorbid conditions. This strategy leads to decreased costs. Besides, outcome measures should be optimized and adequate for these studies as there is variability in outcomes compared to healthy animals (6).

\section{CONCLUSION}

The high prevalence of comorbidities in patients with stroke indicates the need for therapies in preclinical studies that take into account these comorbidities in order to avoid failures in translation to the patient. Preclinical studies are beginning to

\section{REFERENCES}

1. Donnan GA, Fisher M, Macleod M, Davis SM, Royal S, Macleod UKM. Stroke. Lancet. (2018) 371:1612-23. doi: 10.1016/S0140-6736(08)60694-7

2. Karatepe AG, Gunaydin R, Kaya T, Turkmen G. Comorbidity in patients after stroke: Impact on functional outcome. J Rehabil Med. (2008) 40:831-5. doi: 10.2340/16501977-0269

3. O'Donnell MJ, Denis X, Liu L, Zhang H, Chin SL, Rao-Melacini P, et al. Risk factors for ischaemic and intracerebral haemorrhagic stroke in 22 countries (the INTERSTROKE study): A case-control study. Lancet. (2010) 376:112-23. doi: 10.1016/S0140-6736(10)60834-3

4. Soriano-Tárraga C, Giralt-Steinhauer E, Mola-Caminal M, Ois A, RodríguezCampello A, Cuadrado-Godia E, et al. Biological age is a predictor of mortality in ischemic stroke. Sci Rep. (2018) 8:4148. doi: 10.1038/s41598-018-22579-0

5. O'Collins VE, Donnan GA, Macleod MR, Howells DW. Hypertension and experimental stroke therapies. J Cereb Blood Flow Metab. (2013) 33:1141-7. doi: $10.1038 /$ jcbfm.2013.88

6. Ergul A, Hafez S, Fouda A, Fagan SC. Impact of comorbidities on acute injury and recovery in preclinical stroke research: focus on hypertension and diabetes. Transl Stroke Res. (2016) 7:248-60. doi: 10.1007/s12975-016-0464-8

7. Kelly-Cobbs AI, Prakash R, Li W, Pillai B, Hafez S, Coucha M, et al. Targets of vascular protection in acute ischemic stroke differ in evaluate the efficacy of MSC treatment in stroke associated with comorbidities, especially hypertension, for ischemic and hemorrhagic stroke. Regarding aging and diabetes, only ischemic stroke studies have been performed. For the moment, few studies have been performed and contradictory results are being reported. These contradictory results may be due to the use of different stroke and comorbidity models, and to the use of different protocols for administering cell-based therapies. This situation indicates a further need to promote standardization of cell concentration and administration routes. Obesity and hyperglycemia have been completely ignored, although they are frequently present in patients with stroke. For this reason, the role of comorbidities should have a more prominent place in preclinical stroke studies. This will hopefully improve bench-tobedside translation and identify viable therapeutic options.

\section{AUTHOR CONTRIBUTIONS}

FL-G and LD wrote the first draft of the manuscript. MG-dF and LO-O wrote sections of the manuscript. BF, GR-A, and ED$\mathrm{T}$ contributed to manuscript revision and read and approved the submitted version. MG-F contributed to the conception of the study, wrote and revised the manuscript, and approved the submitted version.

\section{ACKNOWLEDGMENTS}

Supported by research grants PI16/01052, INVICTUS PLUS (RD16/0019/0005) (Spanish Neurovascular Network) from the Carlos III Research Institute, Spanish Ministry of Science and Innovation, the European Regional Development Fund (FEDER Funding) and the RESSTORE project (www.resstore.eu), funded by the European Commission under the H2020 program (grant number 681044). We greatly appreciate the support of Morote for editing assistance. type 2 diabetes. Am J Physiol Heart Circ Physiol. (2013) 304:806-15. doi: 10.1152/ajpheart.00720.2012

8. O'Collins VE, Macleod MR, Donnan GA, Horky LL, van der Worp BH, Howells DW. 1,026 experimental treatments in acute stroke. Ann Neurol. (2006) 59:467-77. doi: 10.1002/ana.20741

9. Liu X, Ye R, Yan T, Yu SP, Wei L, Xu G, et al. Cell based therapies for ischemic stroke: from basic science to bedside. Prog Neurobiol. (2014) 115:92-115. doi: 10.1016/j.pneurobio.2013.11.007

10. Marei HE, Hasan A, Rizzi R, Althani A, Afifi N, Cenciarelli C, et al. Potential of stem cell-based therapy for ischemic stroke. Front Neurol. (2018) 9:34. doi: 10.3389/fneur.2018.00034

11. George PM, Steinberg GK. Novel stroke therapeutics: unraveling stroke pathophysiology and its impact on clinical treatments. Neuron. (2015) 87:2973097. doi: 10.1016/j.neuron.2015.05.041

12. Gutiérrez-Fernández M, Rodríguez-Frutos B, Ramos-Cejudo J, VallejoCremades MT, Fuentes B, Cerdán S, et al. Effects of intravenous administration of allogenic bone marrow- and adipose tissue-derived mesenchymal stem cells on functional recovery and brain repair markers in experimental ischemic stroke. Stem Cell Res Ther. (2013) 4:11. doi: $10.1186 /$ scrt159

13. Gutierrez-Fernandez M, Rodríguez-Frutos B, Ramos-Cejudo J, Otero-Ortega L, Fuentes B, Vallejo-Cremades MT, et al. Comparison between xenogeneic 
and allogeneic adipose mesenchymal stem cells in the treatment of acute cerebral infarct: Proof of concept in rats. J Transl Med. (2015) 13:4-13. doi: 10.1186/s12967-015-0406-3

14. Leu S, Lin YC, Yuen CM, Lin YC, Yuen CM, Yen CH, et al. Adiposederived mesenchymal stem cells markedly attenuate brain infarct size and improve neurological function in rats. J Transl Med. (2010) 8:63. doi: 10.1186/1479-5876-8-63

15. Gutiérrez-Fernández M, Rodríguez-Frutos B, Alvarez-Grech J, VallejoCremadesa MT, Expósito-Alcaidea M, Merino J, et al. Functional recovery after hematic administration of allogenic mesenchymal stem cells in acute ischemic stroke in rats. Neuroscience. (2011) 175:394-405. doi: 10.1016/j.neuroscience.2010.11.054

16. Chopp M, Li Y. Treatment of neural injury with marrow stromal cells. Lancet Neurol. (2002) 1:92-100.doi: 10.1016/S1474-4422(02)00040-6

17. Schu S, Nosov M, O’Flynn L, Shaw G, Treacy O, Barry F, et al. Immunogenicity of allogeneic mesenchymal stem cells. J Cell Mol Med. (2012) 16:2094-103. doi: 10.1111/j.1582-4934.2011.01509.x

18. Fisher M, Feuerstein G, Howells DW, Hurn PD, Kent TA, Savitz SI, et al. Update of the stroke therapy academic industry roundtable preclinical recommendations. Stroke. (2009) 40:2244-50. doi: 10.1161/STROKEAHA.108.541128

19. Knoflach M, Matosevic B, Rücker M, Furtner M, Mair A, Wille G, et al. Functional recovery after ischemic stroke - a matter of age: data from the austrian stroke unit registry. Neurology. (2012) 78:279-85. doi: 10.1212/WNL.0b013e31824367ab

20. Bosetti F, Koenig JI, Ayata C, Back SA, Becker K, Broderick JP, et al. Translational stroke research: vision and opportunities. Stroke. (2017) 48:2632-7. doi: 10.1161/STROKEAHA.117.017112

21. Brenneman M, Sharma S, Harting M, Strong R, Cox CS, Aronowski J, et al. Autologous bone marrow mononuclear cells enhance recovery after acute ischemic stroke in young and middle-aged rats. J Cereb Blood Flow Metab. (2010) 30:140-9. doi: 10.1038/jcbfm.2009.198

22. Coelho BP, Giraldi-Guimarães A. Effect of age and gender on recovery after stroke in rats treated with bone marrow mononuclear cells. Neurosci Res. (2014) 88:67-3. doi: 10.1016/j.neures.2014.08.007

23. Zhang L, Yi L, Chopp M, Kramer BC, Romanko M, Gosiewska A, et al. Intravenous administration of human umbilical tissue-derived cells improves neurological function in aged rats after embolic stroke. Cell Transplant. (2013) 22:1569-76. doi: 10.3727/096368912X658674

24. Shen LH, Li Y, Chen J, Cui Y, Zhang C, Kapke A, et al. One-year follow-up after bone marrow stromal cell treatment in middle-aged female rats with stroke. Stroke. (2007) 38:2150-6. doi: 10.1161/STROKEAHA.106.481218

25. Balseanu AT, Buga AM, Catalin B, Wagner DC, Boltze J, Zagrean AM, et al. Multimodal approaches for regenerative stroke therapies: combination of granulocyte colony-stimulating factor with bone marrow mesenchymal stem cells is not superior to G-CSF alone. Front Aging Neurosci. (2014) 6:130. doi: 10.3389/fnagi.2014.00130

26. Buga AM, Scheibe J, Moller K, Ciobanu O, Posel C, Boltze J, et al. Granulocyte colony-stimulating factor and bone marrow mononuclear cells for stroke treatment in the aged brain. Curr Neurovasc Res. (2015) 12:155-62. doi: 10.2174/1567202612666150311112550

27. Ito M, Kuroda S, Sugiyama T, Maruichi K, Kawabori M, Nakayama N, et al. Transplanted bone marrow stromal cells protect neurovascular units and ameliorate brain damage in stroke-prone spontaneously hypertensive rats. Neuropathology. (2012) 32:522-33. doi: 10.1111/j.1440-1789.2011. 01291.x

28. Calió ML, Marinho DS, Ko GM, Ribeiro RR, Carbonel AF, Oyama LM, et al. Transplantation of bone marrow mesenchymal stem cells decreases oxidative stress, apoptosis, and hippocampal damage in brain of a spontaneous stroke model. Free Radic Biol Med. (2014) 70:141-54. doi: 10.1016/j.freeradbiomed.2014.01.024

29. Kranz A, Wagner DC, Kamprad M, Scholz M, Schmidt UR, Nitzsche $\mathrm{F}$, et al. Transplantation of placenta-derived mesenchymal stromal cells upon experimental stroke in rats. Brain Res. (2010) 1315:128-36. doi: 10.1016/j.brainres.2009.12.001

30. Minnerup J, Wagner D-C, Strecker J-K, Pösel C, Sevimli-Abdis S, Schmidt $\mathrm{A}$, et al. Bone marrow-derived mononuclear cells do not exert acute neuroprotection after stroke in spontaneously hypertensive rats. Front Cell Neurosci. (2014) 7:288. doi: 10.3389/fncel.2013.00288

31. Weise G, Lorenz M, Pösel C, Maria Riegelsberger U, Störbeck V, Kamprad M, et al. Transplantation of cryopreserved human umbilical cord blood mononuclear cells does not induce sustained recovery after experimental stroke in spontaneously hypertensive rats.J Cereb Blood Flow Metab. (2014) 34:e1-9. doi: 10.1038/jcbfm.2013.185

32. Pösel C, Scheibe J, Kranz A, Bothe V, Quente E, Fröhlich W, et al. Bone marrow cell transplantation time-dependently abolishes efficacy of granulocyte colony-stimulating factor after stroke in hypertensive rats. Stroke. (2014) 45:2431-7. doi: 10.1161/STROKEAHA.113.004460

33. Taguchi A, Zhu P, Cao F, Kikuchi-Taura A, Kasahara Y, Stern DM, et al. Reduced ischemic brain injury by partial rejuvenation of bone marrow cells in aged rats. J Cereb Blood Flow Metab. (2011) 31:855-67. doi: $10.1038 /$ jcbfm.2010.165

34. Wagner DC, Bojko M, Peters M, Lorenz M, Voigt C, Kaminski A, et al. Impact of age on the efficacy of bone marrow mononuclear cell transplantation in experimental stroke. Exp Transl Stroke Med. (2012) 4:17. doi: 10.1186/2040-7378-4-17

35. Wang C, Fei Y, Xu C, Zhao Y, Pan Y. Bone marrow mesenchymal stem cells ameliorate neurological deficits and blood-brain barrier dysfunction after intracerebral hemorrhage in spontaneously hypertensive rats. Int J Clin Exp Pathol. (2015) 8:4715-24.

36. Ding R, Lin C, Wei S, Zhang N, Tang L, Lin Y, et al. Therapeutic benefits of mesenchymal stromal cells in a rat model of hemoglobininduced hypertensive intracerebral hemorrhage. Mol Cells. (2017) 40:133-42. doi: 10.14348/molcells.2017.2251

37. Yan T, Venkat P, Ye X inchun, Chopp M, Zacharek A, Ning R, et al. HUCBCs increase angiopoietin 1 and induce neurorestorative effects after stroke in T1DM rats. CNS Neurosci Ther. (2014) 20:935-44. doi: 10.1111/cns.12307

38. Cui C, Ye X, Chopp M, Venkat P, Zacharek A, Yan T, et al. miR145 regulates diabetes-bone marrow stromal cell-induced neurorestorative effects in diabetes stroke rats. Stem Cells Transl Med. (2016) 5:1656-67. doi: 10.5966/sctm.2015-0349

39. Chen J, Ye X, Yan T, Zhang C, Yang XP, Cui X, et al. Adverse effects of bone marrow stromal cell treatment of stroke in diabetic rats. Stroke. (2011) 42:3551-8. doi: 10.1161/STROKEAHA.111.627174

40. Yan T, Ye X, Chopp M, Zacharek A, Ning R, Venkat P, et al. Niaspan attenuates the adverse effects of bone marrow stromal cell treatment of stroke in type one diabetic rats. PLOS ONE. (2013) 8: e0081199. doi: 10.1371/journal.pone.0081199

41. Ye X, Yan T, Chopp M, Zacharek A, Ning R, Venkat P, et al. Combination BMSC and Niaspan treatment of stroke enhances white matter remodeling and synaptic protein expression in diabetic rats. Int J Mol Sci. (2013) 14:22221-32. doi: 10.3390/ijms141122221

42. Yan T, Venkat P, Chopp M, Zacharek A, Ning R, Cui Y, et al. Neurorestorative therapy of stroke in type two diabetes rats treated with human umbilical cord blood cells. Stroke. (2015) 46:2599-606. doi: 10.1161/STROKEAHA.115.009870

43. Ding G, Chen J, Chopp M, Li L, Yan T, Li Q, et al. Cell treatment for stroke in type two diabetic rats improves vascular permeability measured by MRI. PLoS ONE. (2016) 11:e0149147. doi: 10.1371/journal.pone.01 49147

44. Hu J, Liu B, Zhao Q, Jin P, Hua F, Zhang Z, et al. Bone marrow stromal cells inhibits HMGB1-mediated inflammation after stroke in type 2 diabetic rats. Neuroscience. (2016) 324:11-9. doi: 10.1016/j.neuroscience.2016.02.058

45. Xiang J, Hu J, Shen T, Liu B, Hua F, Zan K, et al. Bone marrow mesenchymal stem cells-conditioned medium enhances vascular remodeling after stroke in type 2 diabetic rats. Neurosci Lett. (2017) 644:62-6. doi: 10.1016/j.neulet.2017.02.040

46. Yan T, Venkat P, Chopp M, Zacharek A, Ning R, Roberts C, et al. Neurorestorative responses to delayed human mesenchymal stromal cells treatment of stroke in type 2 diabetic rats. Stroke. (2016) 47:2850-8. doi: 10.1161/STROKEAHA.116.014686

47. Leong XF, Ng CY, Jaarin K. Animal models in cardiovascular research: hypertension and atherosclerosis. Biomed Res Int. (2015) 2015:1-7. doi: $10.1155 / 2015 / 528757$ 
48. Diz DI, Baer PG, Nasjletti A. Angiotensin II-induced hypertension in the rat. Effects on the plasma concentration, renal excretion, and tissue release of prostaglandins. J Clin Invest. (1983) 72:466-77. doi: 10.1172/JCI110994

49. Bailey EL, Smith C, Sudlow CL, Wardlaw JM. Is the spontaneously hypertensive stroke prone rat a pertinent model of sub cortical ischemic stroke? A systematic review. Int J Stroke. (2011) 6:434-44. doi: 10.1111/j.1747-4949.2011.00659.x

50. Seshadri S, Beiser A, Kelly-Hayes M, Kase CS, Au R, Kannel WB, et al. The lifetime risk of stroke: estimates from the framingham study. Stroke. (2006)37:345-50. doi: 10.1161/01.STR.0000199613.38911.b2

51. Bruno A, Liebeskind D, Hao Q, Raychev R. Diabetes mellitus, acute hyperglycemia, and ischemic stroke. Curr Treat Options Neurol. (2010) 12:492-503. doi: 10.1007/s11940-010-0093-6

52. Rom S, Zuluaga-Ramirez V, Gajghate S, Seliga A, Winfield M, Heldt NA, et al. Hyperglycemia-driven neuroinflammation compromises BBB leading to memory loss in both diabetes mellitus (DM) Type 1 and Type 2 mouse models. Mol Neurobiol. (2019) 56:1883-96. doi: 10.1007/s12035-018-1195-5

53. Chen R, Ovbiagele B, Feng W. Diabetes and stroke: epidemiology, pathophysiology, pharmaceuticals and outcomes. Am J Med Sci. (2016) 351:380-6. doi: 10.1016/j.amjms.2016.01.011

54. Arboix A, Rivas A, García-Eroles L, de Marcos L, Massons J, Oliveres M. Cerebral infarction in diabetes: clinical pattern, stroke subtypes, and predictors of in-hospital mortality. BMC Neurology. (2005) 5:9. doi: $10.1186 / 1471-2377-5-9$

55. King AJ. The use of animal models in diabetes research. Br J Pharmacol. (2012) 166:877-894. doi: 10.1111/j.1476-5381.2012.01911.x

56. Acharjee S, Ghosh B, Al-Dhubiab BE, Nair AB. Understanding type 1 diabetes: Etiology and models. Can J Diabetes. (2013) 37:269-76. doi: 10.1016/j.jcjd.2013.05.001

57. Sasase T, Ohta T, Masuyama T, Yokoi N, Kakehashi A, Shinohara M. The spontaneously diabetic Torii rat: An animal model of nonobese Type 2 Diabetes with severe diabetic complications. J Diabetes Res. (2013) 2013:1-12. doi: 10.1155/2013/976209

58. Kuwabara WM, Panveloski-Costa AC, Yokota CN, Pereira JN, Filho JM, Torres RP, et al. Comparison of goto-kakizaki rats and high fat diet-induced obese rats: are they reliable models to study type 2 diabetes mellitus? PLoS ONE. (2017) 12:e0189622. doi: 10.1371/journal.pone.0189622

59. Gheibi S, Kashfi K, Ghasemi A. A practical guide for induction of type2 diabetes in rat: Incorporating a high-fat diet and streptozotocin. Biomed Pharmacother. (2017) 95:605-13. doi: 10.1016/j.biopha.2017.08.098

60. Szkudelski T. Streptozotocin-nicotinamide-induced diabetes in the rat. Characteristics of the experimental model. Exp Biol Med. (2012) 237:48190.doi: 10.1258/ebm.2012.011372

61. Lau LH, Lew J, Borschmann K, Thijs V, Ekinci EI. Prevalence of diabetes and its effects on stroke outcomes: a meta-analysis and literature review. J Diabetes Investig. (2018). doi: 10.1111/jdi.12932. [Epub ahead of print].

62. Toni D, De Michele $M$, Fiorelli $M$, Bastianello S, Camerlingo $M$, Sacchetti ML, et al. Influence of hyperglycaemia on infarct size and clinical outcome of acute ischemic stroke patients with intracranial arterial occlusion. J Neurol Sci. (1994) 123:129-33.doi: 10.1016/0022-510X(94)90 214-3

63. Snarska KK, Bachórzewska-Gajewska H, Kapica-Topczewska K, Drozdowski W, Chorazy M, Kułakowska A, et al. Hyperglycemia and diabetes have different impacts on outcome of ischemic and hemorrhagic stroke. Arch Med Sci. (2017) 13:100-8. doi: 10.5114/aoms.2016.61009

64. Fuentes B, Castillo J, San José B, Leira R, Serena J, Vivancos J, et al. The prognostic value of capillary glucose levels in acute stroke:the glycemia in acute stroke (GLIAS) study. Stroke. (2009) 40:562-8. doi: 10.1161/STROKEAHA.108.519926

65. Saha JK, Xia J, Engle SK, Chen YF, Glaesner W, Jakubowski JA. A model of controlled acute hyperglycemia in rats: Effects of insulin and glucagon-like peptide-1 analog. J Pharmacol Exp Ther. (2006) 316:115964.doi: 10.1124/jpet.105.093534

66. Di Filippo C, Marfella R, Cuzzocrea S, Piegari E, Petronella P, Giugliano D, et al. Hyperglycemia in streptozotocin-induced diabetic rat increases infarct size associated with low levels of myocardial HO-1 during ischemia/reperfusion. Diabetes. (2005) 54:803-10. doi: $10.2337 /$ diabetes.54.3.803

67. Suk SH, Sacco RL, Boden-Albala B, Cheun JF, Pittman JG, Elkind MS, et al. Abdominal obesity and risk of ischemic stroke: The Northern Manhattan Stroke Study. Stroke. (2003) 34:1586-92. doi: 10.1161/01.STR.0000075294.98582.2F

68. Strazzullo P, D’Elia L, Cairella G, Garbagnati F, Cappuccio FP, Scalf L. Excess body weight and incidence of stroke: Meta-analysis of prospective studies with 2 million participants. Stroke. (2010) 41:418-26. doi: 10.1161/STROKEAHA.109.576967

69. Yatsuya H, Folsom AR, Yamagishi K, North KE, Brancati FL, Stevens J, et al Race- and sex-specific associations of obesity measures with ischemic stroke incidence in the Atherosclerosis Risk in Communities (ARIC) study. Stroke. (2010) 41:417-25. doi: 10.1161/STROKEAHA.109.566299

70. Pezzini A, Grassi M, Paciaroni M, Zini A, Silvestrelli G, Iacoviello L, et al. Obesity and the risk of intracerebral hemorrhage: The multicenter study on cerebral hemorrhage in Italy. Stroke. (2013) 44:1584-9. doi: 10.1161/STROKEAHA.111.000069

71. Lutz TA, Woods SC. Overview of animal models of obesity. Curr Protoc Pharmacol. (2012) 5.61:1-22. doi: 10.1002/0471141755.ph0561s58

72. Chang KA, Lee JH, Suh YH. Therapeutic potential of human adiposederived stem cells in neurological disorders. J Pharmacol Sci. (2014) 126:293-301. doi: 10.1254/jphs.14R10CP

73. Parker AM, Katz AJ. Adipose-derived stem cells for the regeneration of damaged tissues. Expert Opin Biol Ther. (2006) 6:567-78. doi: $10.1517 / 14712598.6 .6 .567$

74. Ra JC, Shin IS, Kim SH, Kang SK, Kang BC, Lee HY, et al. Safety of intravenous infusion of human adipose tissue-derived mesenchymal stem cells in animals and humans. Stem Cells Dev. (2011) 20:1297-308. doi: 10.1089/scd.2010.0466

75. Gutiérrez-Fernández M, Rodríguez-Frutos B, Otero-Ortega L, Ramos-Cejudo J, Fuentes B, Díez-Tejedor E. Adipose tissue-derived stem cells in stroke treatment: from bench to bedside. Discov Med. (2013) 16:37-43.

76. Manwani B, Liu F, Xu Y, Persky R, Li J, McCullough LD. Functional recovery in aging mice after experimental stroke. Brain Behav Immun. (2011) 25:1689700. doi: 10.1016/j.bbi.2011.06.015

Conflict of Interest Statement: The authors declare that the research was conducted in the absence of any commercial or financial relationships that could be construed as a potential conflict of interest.

Copyright () 2019 Laso-García, Diekhorst, Gómez-de Frutos, Otero-Ortega, Fuentes, Ruiz-Ares, Diez-Tejedor and Gutiérrez-Fernández. This is an open-access article distributed under the terms of the Creative Commons Attribution License (CC BY). The use, distribution or reproduction in other forums is permitted, provided the original author(s) and the copyright owner(s) are credited and that the original publication in this journal is cited, in accordance with accepted academic practice. No use, distribution or reproduction is permitted which does not comply with these terms. 